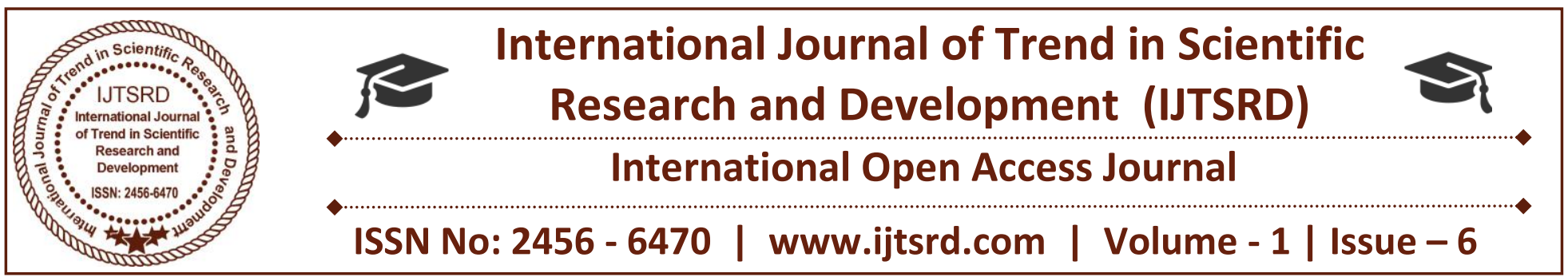

\title{
A Review on Anaerobic Treatment in Decentralized and Source-Separation-Based Sanitation Concepts: Applicability in Developing Countries
}

\author{
Atun RoyChoudhury \\ M.E. Scholar, Department of Civil \\ Engineering, Annamalai University, \\ Annamalainagar, Tamil Nadu, India
}

\author{
Ashok Kumar N \\ Asst. Professor, Department of Civil \\ Engineering, Annamalai University, \\ Annamalainagar, Tamil Nadu, India
}

\author{
Arutchelvan V \\ Professor, Department of Civil Engineering, Annamalai \\ University, Annamalainagar, Tamil Nadu, India
}

\begin{abstract}
Providing reliable and cheap effluent treatment in rural areas may be a challenge in several components of the globe, significantly in developing countries. The issues and limitations of the centralized approaches for effluent treatment square measure increasingly egress. Anaerobic digestion of effluent ought to be a core technology utilized in localized sanitation systems particularly once their objective is

quality depends on the anaerobic effluent quality and native necessities for final effluent quality. Understanding the receiving surroundings is crucial for technology choice and will be accomplished by conducting a comprehensive website analysis method. A spread of technological solutions for the treatment of domestic effluent streams and recycle of resources is mentioned during this paper.
\end{abstract} additionally resource conservation and recycling. The foremost economical system involves separate assortment and anaerobic digestion of the foremost focused domestic effluent streams: black or brown water and solid fraction of room waste. Separate assortment victimization negligible quantity of transport water beside saving this resource permits applying a targeted treatment. A comparatively tiny volume of digestible effluent is directly reused for fertilization or processed once a top quality product is needed. Clean nutrient production needs advanced multi-step treatment, however, the standard of product is unhazardous. The difficulty of organic micropollutants and their accumulation within the surroundings is recently usually self-addressed. Anaerobic treatment of total domestic effluent stream is applied further. Treated during this means effluent is discharged or used for irrigation or fertilization. The post-treatment is sometimes needed and its rate of

Keywords: Anaerobic digestion; Decentralize treatment; Recycling; Sanitation

\section{INTRODUCTION}

Globally, billions of individuals lack access to safe water and adequate sanitation (WHO, 2002; Ho, 2003). Regarding $40 \%$ of the world's population lacks basic sanitation and sanitation coverage is often abundant lower in rural areas than in urban areas (WHO, 2002). Estimates of the globe Health Organization (WHO) and also the facility and Sanitation cooperative Council indicate that $25 \%$ of the developing country urban dwellers lack access to sanitation services with a far higher proportion of the agricultural populations of developing countries reaching up to $82 \%$ (CNES, 2003). The dearth of adequate sanitation services results in many diseases (Fig. 1). The WHO estimates that a pair of.1 million 
folks die annually from regular diseases (WHO, 2002). Worldwide, an important development has been created in effluent treatment for urban areas as compared to rural areas that lag so much behind. Effluent treatment plants represent one among the key investments thanks to high price cost of capital opportunity cost $\}$ additionally to operation and maintenance cost. Restricted native budgets, lack of native experience, and lack of funding ended in an inadequate operation of effluent treatment plants in developing countries (Paraskevas et al., 2002). Moreover, little and isolated villages or settlements with low population densities will be served by localised systems that square measure less complicated and efficient (Butler and MacCormick, 1996; Otterpohl et al., 1997; Hedberg, 1999; Wilderer and Schreff, 2000; Paraskevas et al., 2002; USEPA, 2005). The big capital investment of sewerage system and pumping prices related to centralized systems will be reduced, so increasing the affordability of effluent management systems. The dearth of analysis and development activities in developing countries results in the choice of inappropriate technology in terms of the native environmental condition and physical conditions, monetary and human resource capabilities, and social or cultural satisfactoriness. Suburbanized, property sanitation ideas specialize in treatment and utilization of resources gift in the domestic effluent. 3 main resources square measure these days considered: bio-energy generated from the transformation of organic material, plant nutrients (nitrogen and phosphorus as main nutrients however additionally $\mathrm{K}$ and sulphur) and water (produced when advanced treatment of cleaner effluent streams). Treatment of effluent streams is chosen in such the simplest way that their employment potential is preserved. Each focused and fewer focused waste (water) streams square measure made in a very minute way for blackwater (faeces and urine) and gray water (originating from the shower, bath, laundry).

\section{Most Common Localized Treatment and Disposal Ways}

\subsection{Primary Treatment Ways}

There area unit many onsite waste product treatment systems that are designed, created, operated and maintained properly can give adequate service and health advantages. The straightforward tank system is that the most typically celebrated primary treatment methodology for onsite waste product treatment owing to its sizeable blessings. Septic tanks take away most settleable solids and performance as Associate in nursing anaerobic bioreactor that promotes partial digestion of organic matter. Their main reason for failure is that the unsuitableness of the soil and therefore the website characteristics (Les and Ashantha, 2003). The Imhoff tank is another primary treatment methodology which will accommodate higher flow rates than the tank, however, it's less common. Each systems area unit cheap and easy to work and maintain. Yet, sludge could cause Associate in Nursing odor downside if unbroken untreated for a protracted time. The standard onsite waste product treatment systems aren't effective in removing nitrate and phosphorus compounds and reducing morbific organisms. As such, these systems are often used before more treatment and disposal. the straightforward tank system may be changed to produce the advanced primary treatment of the waste product. The results of the modification would be a tank with Associate in nursing effluent filter vault or a tank with hooked up growth. The filter is that the extra element for the previous tank. This filter prevents some solids from coming into the effluent and consequently preventive the treatment system as a full (USEPA, 2002). As for the latter, it's in the main Associate in nursing aerobic system used wherever the quality anaerobic septic tanks aren't an honest possibility. They're primarily employed in places wherever the soil is poor, the groundwater is high, the land accessible is tiny or the positioning is sensitive.

\subsection{Secondary Treatment Ways}

Many secondary treatment ways exist for localized waste product treatment; every having blessings and downsides (Table 2). Considering that sand is that the commonest and accessible media for filters, typically media filter is like a sand filter. Generally, in areas with deep, leaky soils, septic tank-soil absorption systems are often used. On the opposite hand, in areas with shallow, terribly slowly leaky or extremely leaky soils a lot of difficult onsite systems are needed.

\subsection{Treatment/Disposal Ways}

Disposal ways are often easy disposal ways akin to the evaporation and evapotranspiration, surface water discharge and recycle. They will even be treatment and disposal ways at the same time akin to the subterraneous waste product infiltration, the land application and therefore the created wetlands. The assorted treatment/ disposal ways give extra treatment to the waste product before the ultimate disposal. An 
outline of the foremost widespread disposal ways is represented in Fig. 3. Given the acceptable website conditions, subterraneous soil absorption is sometimes the simplest methodology of waste product disposal for single dwellings owing to its simplicity, stability, and low value. There area unit many sorts of subterraneous soil absorption systems (USEPA, 2002). Trenches and beds, oozing pits, mounds, and fills area unit all lined excavations crammed with porous media with a way for introducing and distributing the waste product throughout the system (USEPA, 2002). Subterraneous waste product infiltration systems could also be the simplest various for sites with acceptable soil conditions, groundwater characteristics, slopes and alternative options. The trenches and beds will operate effectively in most climates, don't would like electricity for operation and area unit less expensive than the opposite systems of subterraneous waste product infiltration. However, they can't be employed in areas with extremely leaky soil.

\subsection{Anaerobic Treatment}

Anaerobic digestion of waste product may be a property possibility as recovery of energy is applied whereas nutrients area unit preserved for recycle. The appliance of anaerobic digestion of domestic waste product has been restricted to tropical countries wherever large-scale plants are or area unit below construction (Hulshof-Pol et al. 1997) in centralized ideas. Results of recent analysis on digestion of waste material below lower temperature conditions (e.g. Elmitwalli 2000; Mahmoud 2002; Seghezzo 2004) and skill from industrial sector proves an oversized potential of anaerobic treatment for the total domestic waste product or separated targeted streams. Despite assorted blessings of anaerobic treatment (Lettinga et al. 2001), typically it doesn't manufacture effluents which will adjust to the standards for recycling in agriculture or discharge to the setting. So posttreatment are in most cases needed.

Anaerobic treatment of total waste product typically it's unimaginable to produce regional sewerage facilities because of socio-economic constraints. In several things total domestic waste material is collected and transported to a central place within the community wherever it's discharged once treatment if any. On-the-scene solutions, whether or not house onsite, the community on website or combination of each area unit typically the sole choice to improve sanitation in these regions or, sadly in several cases, to introduce sanitation. Assortment and treatment of whole waste product mixture aren't perfect various, in light-weight of earlier issues, to realize associate in nursing optimised treatment and highest recovery of resources in one. Nutrients area unit diluted in giant waste product volumes and their recovery is economically less possible. Energy recovery and recycle from moderate targeted in terms of organic matter total waste product. The stream is additionally less enticing than just in case of the extremely targeted medium. Still, however, by Associate in a Nursing acceptable combination of technologies property solutions are often applied. Water inadequacy will increase the chance for native reclamation/reuse solutions. The utilization of anaerobic treatment completed by post-treatment techniques offers the efficient methodology for reclaiming domestic waste product and nutrients for agricultural production. An unremarkably used onthe-scene system to (pre)treat the complete waste product may be a tank followed in some cases by soil absorption (U.S. EPA 1980, 2000b). Normally very little attention has been paid to the planning improvement of those systems typically operative below sub-optimal temperature conditions. The processes occurring in septic tanks are sinking of suspended matter, anaerobic conversion of organic matter and accumulation of inert particles. As a result of the horizontal flow of the incoming waste product stream, no contact between sludge and waste product is established, ensuring at low conversion of dissolved elements. At the all-time low of the tank, sludge accumulates and forms a sludge bed, reducing internet volume and thence the waste product hydraulic retention time (HRT). Before the potency of sinking deteriorates and particles area unit washed out of the reactor the sludge has to be removed. Oil, fat, and alternative floating materials type a scum layer on the surface. Up to five hundredth of organic matter, reckoning on temperature and solids retention time (SRT), decomposes, whereas the rest should be removed sporadically by pumping from the tank (EPA 2000a). Tanks need pumping at frequent intervals to avoid reduction of the effective volumetrical capability. The frequency of tank desludging depends on sludge and scum accumulation rates and typically varies between half and a number of other years. 

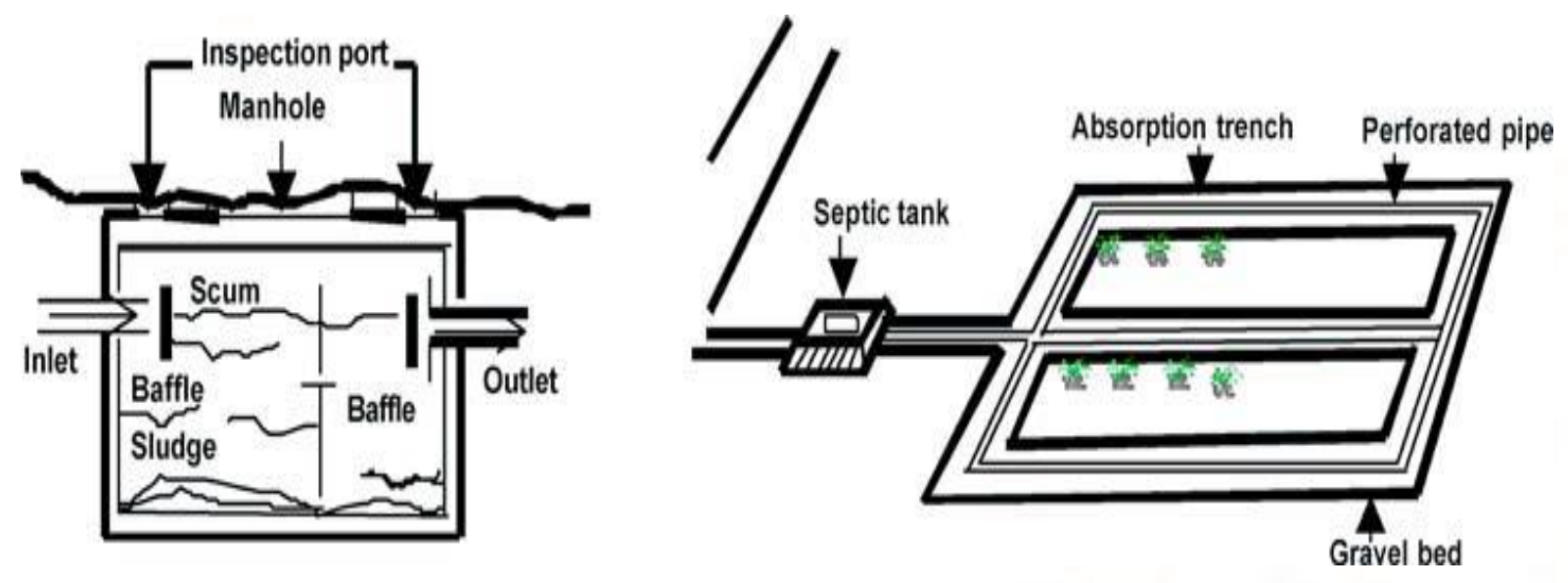

Figure 1: ancient treatment facilities employed in unsewered regions and for collective treatment of waste product from little populations: tank followed or not by absorption (filtration) field

\subsection{Choosing a technology}

Choosing the "Most acceptable Technology" isn't a straightforward task however it might scale back the danger of future issues and failures. The two key problems in selecting a treatment technology are area unit affordability and appropriateness (Grau, 1996). Affordability relates to the economic conditions of the community whereas appropriateness relates to the environmental and social conditions.

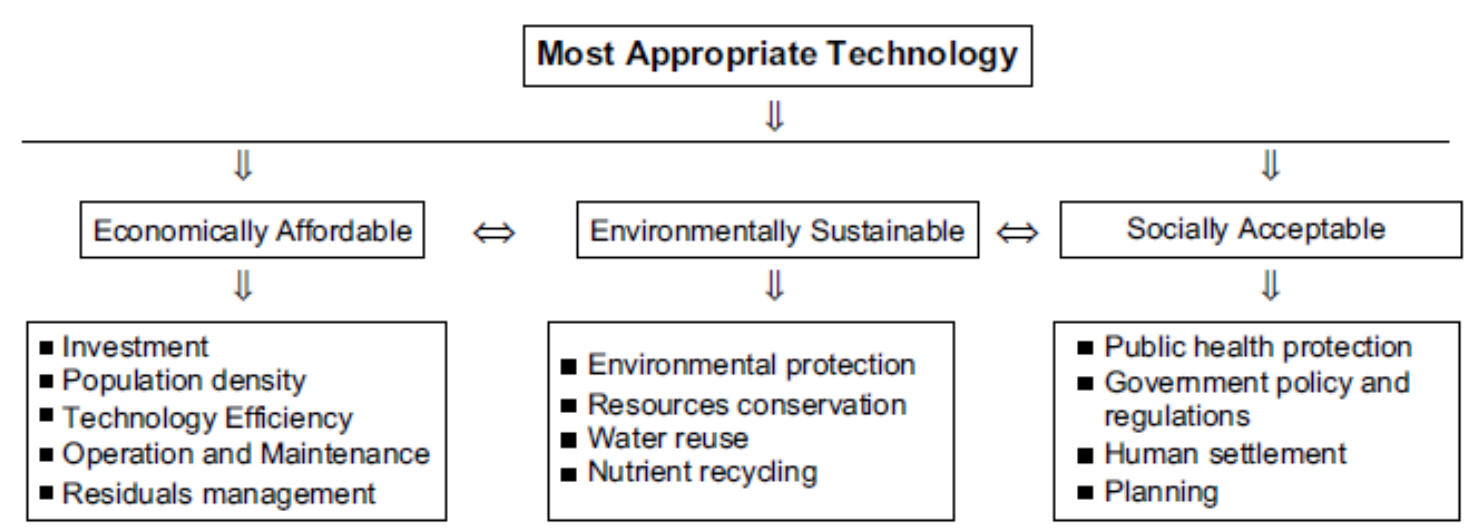

Figure 2: Characteristics of the foremost acceptable Technology

\subsection{Post-Treatment of Anaerobic Effluent}

Because of not complete degradation of organic matter in anaerobic reactors, there'll be perpetually a fraction of remaining COD gift within the effluent, next to morbific organisms and nutrients. The selection of a post-treatment depends powerfully on the characteristics of the anaerobic effluent and on native standards set by authorities for recycling of treated effluent or discharge to the setting. In Europe, anaerobic waste material treatment usually has got to adjust to COD discharge standards established by the council Directive 91/271/EEC on Urban waste product Treatment (European Council of Ministers 1991, below a hundred $25 \mathrm{mgCOD} / \mathrm{L})$. For unrestricted irrigation, the globe Health Organisation (WHO one989) set the standards of below one thousand soiled coliforms in one hundred metric capacity unit and fewer than 1 parasitic worm egg per 1 treated waste product.

\section{7 'Clean'" nutrient production}

Direct recycle of anaerobically treated effluent are typically unimaginable because of a hygienical risk. 
Posttreatment are then needed to get rid of morbific organisms. Additionally as a result of energy costs tend perpetually to grow and agriculture is powerfully passionate about fossil fuels for the production of $\mathrm{N}$ and $\mathrm{P}$ fertilizers, the recycle of "clean" $\mathrm{N}$ and $\mathrm{P}$ can become attention-grabbing (Helsel 1992; Gajdos 1998; Verstraete, et al. 2004). For clean nutrient production, advanced technologies area unit needed. Recovery of "clean" nutrients from an anaerobically treated targeted waste product is, next to biogas, a possible supply of revenue, partly counteractive the prices of treatment. Though each recovery and removal techniques are investigated or applied in an exceedingly full-scale, restricted info is offered to choose that route, removal or recovery, ought to be taken in supply separation sanitation ideas. Industrial ammonia production for fertilizer business remains fairly cheap. Atomic number 7 is additionally not a finite mineral and energy needed for recovery is commonly capable the combined energy needed for biological $\mathrm{N}$ removal (for instance via a combined SHARON/Anammox process) and industrial ammonia production (Maurer et al. 2003a, b; Wilsenach et al. 2003). Additionally with a high load of ammonia created through husbandry and therefore the lack of farmland close to cities, being a case in several countries, economical atomic number 7 removal from one by one collected black water might sway be a lot of possible possibility than recovery.

\subsection{Nutrient Recovery}

Stripping atomic number 7 within the effluent of associate in nursing anaerobic reactor treating black water is especially gifted as ammonium ion. By raising the hydrogen ion concentration the ammonium ion is born-again to without delay soluble ammonia. In touch with the vaporific part, ammonia is transferred from the water to the gas part. In remotion towers applied for ammonia remotion, the water and gas flow count-currently and a high contact surface are ensured by the presence of stuff. 2 sorts of processes area unit distinguished: air- and steam remotion, differing within the finish treatment of the ammonia made gas. Within the air remotion, a method the ammonia made air is either clean with acid or combusted. Throughout the steam remotion, liquid ammonia (salt) is created, which might be targeted by reflux (Janus \&amp; van Delaware Roest 1997).

\subsection{Issues of Concern in Developing Countries}

Often, the high value of wastewater treatment and management may be a major impediment towards implementing such comes. Governments in developing countries have a lot of pressing wants than waste product management akin to coping with war and conflicts, health care and food offer. Waste product management is often low on the list of priorities. Several developing countries suffer from political interference in environmental choices akin to website choice and alternative aspects involving construction and operation. Even the foremost advanced technology ought to be supported by the suitable establishments and enforced legislation to confirm most potency. The resource of international organizations and developed countries is important, nevertheless, it's imperative that native conditions area unit thought-about form full use of any aid. Otherwise, there's no purpose of funding such comes. The adoption of inappropriate technology and failure to require into thought the native conditions of the targeted community lead to project failure that's typically damn on the shortage of technical knowledge and monetary resources. Typically millions area unit spent on construction and a number of greenbacks on gathering reliable style information. Replication of prospering comes is helpful however the system ought to be adjusted to the native conditions, particularly weather conditions. a lot of typically than not, the affordable technology is chosen with none alternative thought. Rural areas in developing countries cannot meet current and future sanitation needs with only one funded project. A comprehensive and semipermanent strategy that needs intensive designing and implementation phases is significant for property waste product management.

\section{DISCUSSION}

The prime objective of decentralized sanitation ideas is to guard the surroundings and public health. However, by combining acceptable technologies, economical treatment and generation of reusable resources are obtained. The selection of a technological theme depends on native circumstances and needs. in a very new location to be engineered or in a very scenario once there's no sanitation infrastructure in any respect the most recovery and reprocessing of resources is achieved once waste matter streams of a unique degree of pollution is singly collected and definitively treated. Blackwater is then singly collected from the gray water. Additional 
separation could involve an assortment of sewage and brown water. The treatment is house-on-site, community-on-site or combination of each. Once reprocess is Associate in the Nursing objective, a negligible quantity of transport water ought to be used for black water.

\section{CONCLUSION}

Traditionally anaerobic treatment of domestic effluent was applied in redistributed sanitation. Alone, it doesn't give an enough degree of effluent purification and resource apply isn't enforced. Aggregation storage tank effluent and transporting it employing a tiny bore facility to a semi-central post-treatment unit leads to a major improvement of redistributed sanitation. If Associate in nursing economical post-treatment is applied, treated effluent is used for irrigation or discharged to surface water. For total domestic effluent stream additional advanced, high rate anaerobic reactors followed by post-treatment is applied leading to a demanded effluent quality for irrigation, fertilization or discharge. The introduction of pee separation considerably improves the nutrient apply potential and property of the native sanitation. Supply separation of effluent streams and their affinitive treatment allows targeted treatment and the most recovery of resources. Effluent containing the best organic and nutrient load, black water and optionally room waste, is ideally digestible during an easy reactor configuration. Betting on native necessities, anaerobic digestion method potency direct applies of the digestible medium is applied. Once the applicable standards are tight, a multi-step advanced treatment method leading to a clean nutrient production and/or removal is applied. The way of effluent assortment and implementation scale (houseon-site or community-on-site) confirm form of the treatment system to be designated.

\section{REFERENCES}

1) Butler, R., MacCormick, T., 1996. Opportunities for decentralized treatment, sewer mining, and effluent reuse. Desalination 106, 273-283.

2) CNES (Citizen Network on Essential Services), 2003. Approaches to Sanitation Services. Water Policy Series A. Water and Domestic Policy Issues A5, 12.

3) Elmitwalli TA (2000) Anaerobic treatment of domestic sewage at low temperature. PhD Thesis, Sub-department of Environmental Technology, Wageningen University.
4) Gajdos R (1998) Bioconversion of organic waste by the year 2010: to recycle elements and save energy. Resources Conserv. Recycling 23: 67-86.

5) Hedberg, T., 1999. Attitudes to traditional and alternative sustainable sanitary systems. Water Science and Technology 39 (5), 9-16.

6) Helsel ZR (1992) Energy in farm production In: Fluck RC (Eds.) Energy in World Agriculture (pp 177-201). vol. 6 Elsevier, New York.

7) Ho, G., 2003. Small water and wastewater systems: pathways to sustainable development? Water Science and Technology 48 (11-12), 7-14.

8) [8] Hulshof-Pol L, Euler H, Eitner A \& Grohantz TBW (1997) State of the art, sector review. Anaerobic treands. WQI July/August, 1997, 3133.

9) Janus HM \& van der Roest H (1997) Don't reject idea of treating reject water. Water Sci. Tech. 35(10): 27-34.

10) Les, D., Ashantha, G., 2003. An investigation into the role of site and soil characteristics in on-site sewage treatment. Environmental Geology 44 (4), 467-477.

11) Lettinga $G$, Lier van JB \& Zeeman G (2001) Sustainability in environmental protection, centralisation or decentralisation in Urban sanitation. Presented on the international symposium on low cost wastewater treatment and reuse, February, 3-4, 2001, Cairo, Egypt.

12) Mahmoud N (2002) Anaerobic pre-treatment of sewage under low temperature $\left(15 \_\mathrm{C}\right)$ conditions in an integrated UASBDigester system. PhD thesis of Wageningen University.

13) Maurer M, Schwegler P \& Larsen TA (2003b) Nutrients in urine: energetic aspects of removal and recovery. Water Sci. Tech. 48(1): 37-46.

14) Otterpohl, R., Grottker, M., Lange, J., 1997. Sustainable water and waste management in urban areas. Water Science and Technology 35 (9), 121133. 
15) Paraskevas, P.A., Giokas, D.L., Lekkas, T.D., 2002. Wastewater management in coastal urban areas: the case of Greece. Water Science and Technology 46 (8), 177-186.

16) Seghezzo L (2004) Anaerobic treatment of domestic wastewater in subtropical regions. $\mathrm{PhD}$ thesis of Wageningen University.

17) USEPA (U.S. Environmental Protection Agency), 2002. On-site Wastewater Treatment Systems Manual. EPA/625/R-00/008. Office of Water and Office of Research and Development, Washington, DC.

18) USEPA (United States Environmental Protection Agency), 2005. Handbook for Managing Onsite and Clustered (Decentralized) Wastewater Treatment Systems, EPA/832-B-05-001. Office of Water, Washington, DC, 66 pp.

19) Verstraete W, Morgan-Sagastume F, Aiyuk S, Waweru M, Rabaey K \& Lissens G (2004) Anaerobic digestion as a core technology is sustainable management of organic matter. Presented on the 10th Anaerobic Digestion Congress, Montreal, Canada, Aug-Sep, 2004.

20) WHO (World Health Organization), 2002. Environmental Health. Eastern Mediterranean Regional Center for Environmental Health Activities (CEHA).

21) Wilderer, P.A., Schreff, D., 2000. Decentralized and centralized wastewater management: a challenge for technology developers. Water Science and Technology 41 (1), 1-8. 